\title{
RETRACTED ARTICLE: Vertebroplasty: an international point of view on this "minimally invasive" surgical technique
}

\author{
B. Saccomanni
}

Received: 18 April 2011 / Accepted: 9 August 2011 /Published online: 8 December 2011

(C) International Osteoporosis Foundation and National Osteoporosis Foundation 2011

This article has been withdrawn due to plagiarism. The original work is: Surgery: Vertebroplasty: one solution does not fit all. Gunnar B. J. Andersson: Nature Reviews Rheumatology 5, 662-663 (December 2009) doi:10.1038/ nrrheum.2009.233.

This article has been withdrawn due to plagiarism. The original work is: Surgery: Vertebroplasty: one solution does not fit all. Gunnar B. J. Andersson: Nature Reviews Rheumatology 5, 662-663 (December 2009) doi:10.1038/nrrheum.2009.233.

B. Saccomanni $(\bowtie)$

Orthopaedic and Trauma Surgery, ASL Bari,

viale Regina Margherita, 74,

70022, Altamura, BA, Italy

e-mail: bernasacco@yahoo.it

B. Saccomanni

Ambulatorio di Ortopedia e Traumatologia,

Via della Conciliazione, 65 ,

74014, Laterza, TA, Italy 